\title{
Inclusive Action Game Presenting Real-time Multimodal Presentations for Sighted and Blind Persons
}

\author{
Masaki Matsuo* \\ m.matsuo@aist.go.jp \\ National Institute of Advanced \\ Industrial Science and Technology \\ (AIST) \\ Kashiwa, Japan \\ Atsushi Katagiri \\ Push-pop Co. Ltd. \\ Kumagaya, Japan
}

\author{
Takahiro Miura ${ }^{* \dagger}$ \\ miura-t@aist.go.jp \\ National Institute of Advanced \\ Industrial Science and Technology \\ (AIST) \\ Kashiwa, Japan \\ Masatsugu Sakajiri \\ Tsukuba University of Technology \\ Tsukuba, Japan
}

\author{
Ken-ichiro Yabu ${ }^{\dagger}$ \\ Research Center for Advanced \\ Science and Technology (RCAST), \\ The University of Tokyo \\ Tokyo, Japan
}

\author{
Junji Onishi \\ Tsukuba University of Technology \\ Tsukuba, Japan
}

\author{
Takeshi Kurata \\ National Institute of Advanced \\ Industrial Science and Technology \\ Kashiwa, Japan
}

\author{
Tohru Ifukube ${ }^{\dagger}$ \\ Research Center for Advanced \\ Science and Technology (RCAST), \\ The University of Tokyo \\ Tokyo, Japan
}

\begin{abstract}
Several audio games have been developed for visually impaired players to enjoy only by using audio information. However, sighted persons find it challenging to play these games because they mainly use visual information. To bridge the gap between them, developing games that appropriately present multisensory modalities: To devise an auditory and tactile presentation method that allows the sighted to manipulate and identify features, enabling them to play without visual information. Therefore, we develop an inclusive game that both sighted and visually impaired people can play. Here, we developed a real-time sensory substitution method using auditory and tactile senses, including a tactile game controller (TactCon). Then, we implemented the method in a 2D side-scrolling action game and evaluated the game by either sighted or visually impaired people at a two-month exhibition: not a controlled experiment. Results show that immediate auditory and tactile presentation can be important for visually impaired and sighted gamers to play action games requiring real-time response. Furthermore, we considered how the presentation of moving objects can be understood in an inclusive action game.
\end{abstract}

\footnotetext{
${ }^{*}$ Both authors contributed equally to this research.

${ }^{\dagger}$ Also with Institute of Gerontology (IOG), The University of Tokyo.
}

Permission to make digital or hard copies of all or part of this work for personal or classroom use is granted without fee provided that copies are not made or distributed for profit or commercial advantage and that copies bear this notice and the full citation on the first page. Copyrights for components of this work owned by others than ACM must be honored. Abstracting with credit is permitted. To copy otherwise, or republish, to post on servers or to redistribute to lists, requires prior specific permission and/or a fee. Request permissions from permissions@acm.org.

ICMI '21, October 18-22, 2021, Montréal, Canada

(C) 2021 Association for Computing Machinery.

ACM ISBN 978-1-4503-8481-0/21/10 \$ \$15.00

https://doi.org/10.1145/3462244.3479912

\section{CCS CONCEPTS}

- Human-centered computing $\rightarrow$ Empirical studies in $\mathrm{HCI}$; Field studies; Haptic devices; Auditory feedback; • Applied computing $\rightarrow$ Computer games; • Social and professional topics $\rightarrow$ People with disabilities.

\section{KEYWORDS}

Inclusive game; visual impairments; game accessibility; tactile game controller; multimodal presentation

\section{ACM Reference Format:}

Masaki Matsuo, Takahiro Miura, Ken-ichiro Yabu, Atsushi Katagiri, Masatsugu Sakajiri, Junji Onishi, Takeshi Kurata, and Tohru Ifukube. 2021. Inclusive Action Game Presenting Real-time Multimodal Presentations for Sighted and Blind Persons. In Proceedings of the 2021 International Conference on Multimodal Interaction (ICMI '21), October 18-22, 2021, Montréal, Canada. ACM, New York, NY, USA, 9 pages. https://doi.org/10.1145/3462244.3479912

\section{INTRODUCTION}

The computer game market has recently grown worldwide and will rapidly expand even in the near future [5, 7, 42]. Also, the demand for computer games has tremendously increased following the global COVID-19 pandemic, "stay at home" and social distancing orders issued in many countries [42].

Thus, computer games have become increasingly diversifiable with improvements in hardware performance and software functions. Regardless of stationary and mobile game machines, the screen display density and resolution become higher, and the information that players must grasp visually became larger. However, these upgrades make most enjoyable games difficult for visually impaired people regardless of the various accessibility studies on content and interfaces $[14,27,37,47,48]$. Consequently, visually impaired volunteers have shared information on games they consider playable or unplayable on various game review websites $[1,2,6]$. 
The existence of these sites itself suggests that not many games can be played by visually impaired gamers.

Volunteer developers with visual impairments have developed games for the visually impaired; namely audio games, in which they control game characters using only auditory information, such as sound effects. These audio games present displayed texts on the screen via a synthesized voice using a text-to-speech (TTS) engine and provide other displayed information with alternative sound effects. A game review website called AudioGames.net [2] has accumulated outlines and details of these games, and visually impaired gamers are eager to review and discuss them. However, these games are difficult for sighted gamers, who primarily rely on visual inputs, to play audio games. Thus, there remains a gap between the visually impaired and sighted gamers.

To bridge this gap, several inclusive games are being developed for players to enjoy regardless of their disabilities [25, 32, 33, 38]. Morelli et al. designed the interactions of the bowling and tennis games for visually impaired gamers corresponding to those of sighted gamers on the Nintendo Wii. Also, they proposed exergames for visually impaired gamers. They developed a scheme such that sighted and visually impaired players can move their bodies commonly, and auditory and haptic feedback can substitute for visual feedback. Matsuo et al. developed an accessible action role-playing game (RPG) that anyone can play, regardless of visual conditions [23, 25]. The RPG was designed so players played using only auditory and tactile cues, and graphical features were added to allow sighted players to play it.

However, though they developed the game system, they did not investigate the conditions for inclusive games in detail. Few studies on auditory and tactile substitution techniques of visual information for visually impaired players exist in numerous games with immediate controller operations unrelated to entire body movements. The requirements for sighted gamers playing games via auditory and tactile cues without visual information remain unclear. Understanding these issues can lead to the development of inclusive games for the visually impaired and sighted gamers, thereby resolving the gap in the gaming community. Additionally, it can contribute to the mutual understanding of the visually impaired by the sighted, and vice versa, leading to the realization of an inclusive society.

In this paper, our goal is to investigate the requirements for an inclusive action game with an immediacy that both visually impaired and sighted players control using a gamepad. First, we developed a $2 \mathrm{D}$ side-scrolling action game, enabling gamers to manipulate a player character with auditory and tactile feedback. Furthermore, we developed a novel tactile game controller TactCon, enabling players to acquire real-time tactile feedback without interfering with the controller operation. Then, a two-month field evaluation of the game was conducted at an exhibition for collecting the play logs and impressions by visually impaired and sighted people.

Our research questions are as follows.

Q1. Does an interface enabling simultaneous perception of tactile information and manipulation, enhance gameplay that requires immediacy in visually impaired people?
Q2. Is it possible for sighted individuals to play an action game using only auditory and haptic feedback? If so, what interaction elements are required and what are the characteristics of players who can complete the game?

Q3. What are the elements that make a game inclusive? Specifically, what are the common helping factors play for both people with or without visual impairments?

\section{RELATED WORK}

\subsection{Content, design, and accessibility of games for people with visual impairments}

With the expansion of the computer game market, game review sites for visually impaired gamers have been growing $[1,2]$. The Audiogames.net mainly collects information on audio game content and reviews and has user communities of various games. The Ablegamers.org provides a service to construct an environment that allows visually impaired gamers to play various games, including audio and multisensory games. Beeston et al. investigated the contents of the site and reported several characteristics of gamers with disabilities and the games they played [12]. They reported that most of the people with disabilities had disabilities in the upper and lower limbs. Also, many disabled players used customized controllers and alternative mice on a personal computer [12]. In the Asia-Pacific region, accounting for about half of the gaming market in 2020 [5], the Japanese visually impaired gaming community aggregates game reviews mainly in two sites [1,6]. One deals with inclusive games and the other with audio games. In the former, visually impaired volunteers aggregate reviews about playable games due to superior multimodal sensory feedback. The existence of such a gaming community whose members play games designed for the sighted suggests the possibility of bridging the gap between the communities of gamers with or without visual impairments.

Additionally, various researchers have continually studied game accessibility. Archambault et al. and Meisenberger et al. summarized the requirements of game accessibility, usability, and interfaces for various disabilities [11, 27]. Porter et al. conducted an interview survey on accessibility in the game industry and gamers with disabilities [37]. They reported the diverse issues associated with individuals having disabilities and the problems in the game industry, including the cost to secure accessibility, especially for gamers with disabilities. Zahand describes accessibility requirements for game developers while considering the business values and reasonable accommodations on game design for gamers with disabilities [48]. For game accessibility for visually impaired gamers, Yuan et al. compiled details on audio and other games [47]. Additionally, British Broadcasting Corporation (BBC) has published its accessible games standards [3] and game accessibility guidelines (GAG) [4] based on the standards. However, with the increasing multimedia contents on the web, the World Wide Web consortium (W3C) updated its web content accessibility guidelines (WCAG) as WCAG 2.1 in 2018 [8]. Westin et al. compared GAG and WCAG and reported that GAG is the best practice-oriented and more game-specific [44].

Game accessibility for people with disabilities can be improved with the development of mainstream games. Moreover, Andrade et al. proposed a game design framework that visually impaired 
gamers participate in $[9,10]$, suggesting a continuous increase in the number of visually impaired gamers in the coming years.

\subsection{Audio and inclusive games}

Research on the operation and presentation interfaces is active in realizing games for people with disabilities. For example, the GazePlay is a gaze-based alternative augmentative communication (AAC) package for gamers with multiple physical disabilities [39]. Gerling et al. reported the design flow examples for a game operated by wheelchair movements. $[15,16]$.

Alternatively, several systems and games using 3D sound have been proposed for the blind [19, 36, 41, 43], who have unique abilities in sound source localization and spatial perception [22, 29, 31, $40]$. Honda et al. proposed a game called the BBBeat as a training system for sound source localization using 3D sound [19]. Seki et al. designed the wide-range auditory orientation-training system (WRAOTS) with incorporated game characteristics so that instructors (walking trainers) for the visually impaired enable trainees to develop the auditory sense of simulated actual cases [41]. ShadowRine, developed by Matsuo et al., is an inclusive, action role-playing game that supports three presentation modalities: visual, auditory, and tactile [23, 25]. Notably, they created a game development environment for the blind that uses Otonove for creating text information and an audible mapper for 2D mapping. Using these tools, they also prototyped an inclusive action game and proposed the evaluation scheme for the gameplay and interface using item response theory $[24,30]$.

Moreover, the widespread use of devices that acquire body movements, such as Wiimote and Kinect, exergames has increasingly enabled visually impaired people to play [32, 33, 38]. Morelli et al. developed a series of exergames, such as bowling and tennis, for the blind using feedbacks to tactile and auditory senses and the Wiimote. Rector et al. developed an application called Eyes-free Yoga using Kinect that allows visually impaired people to enjoy yoga. Morelli et al. reported cases where the visually impaired play sports games by identifying tactile feedback to their whole body using physical movements [34].

\subsection{Issues in this study}

The visually impaired gaming environment has been improved, and it is expected that the number of visually impaired gamers will increase. However, no accessibility standards have been established for games that require immediate actions, and few studies are examining effective accessibility measures for such content. Furthermore, most of the inclusive games playable by people with and without disabilities use physical movements, like exergame, and not the traditional gamepad format. To make audio games playable for sighted people, the information presentation conditions for inclusive games must be clarified. Therefore, we formulated the research questions described in the previous section.

\section{IMPLEMENTING INCLUSIVE GAME}

From the perspective of inclusive research proposed by Nind [35], an author with blindness, and other visually impaired gamers involved in the research and development of games reflecting the latent needs of the visually impaired.

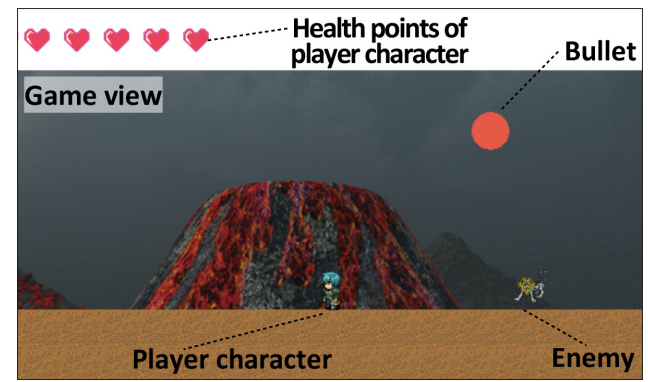

Figure 1: Screenshot of our inclusive side-scroller game

\subsection{Overview}

Fig. 1 shows the overview of the implemented inclusive game. It is an inclusive side-scrolling action game on Windows that sighted and visually impaired individuals can play with typical gamepads and specialized devices. Sighted gamers control a game character mainly by watching the behavior on the screen, whereas the visually impaired manipulate a game character using auditory and/or tactile feedback, as shown in Fig. 2. The game requires that users recognize and judge the current activity in the game and operate the game character as quickly and precisely as possible.

The primary control of this game is similar to various sidescrolling games: moving the player character left or right while using jump actions to aim at the goal and using weapons to beat down enemies along several stages of the game. The stages have obstacles, including walls, pits, and enemy characters. When the character falls into a pit, the player must restart from the corresponding respawning point, usually located near the left-back of the pit. Furthermore, several gimmicks were prepared, including footholds that move regularly, and rocks that decrease the health points of the character riding on them.

\subsection{Play styles and interfaces}

This game contains four play styles: visual, audio, touch, and multimodal plays. The visual play style is mainly for sighted people and those with low vision, and allows the player to play the game by watching the visuals on the screen. The audio play style is designed for blind gamers and is played using sound information from headphones. The touch play style allows players to rely on information from the tactile display. The multimodal play style combines the play styles of the other three modes; the player can use visual, auditory, and tactile information to play.

3.2.1 Audio play. This play style presents information on the screen using several sound effects through headphones. Sound effects corresponding to the game events, include the movement and action sounds of the player character, sounds when the character hits the enemies, and decreasing the sounds of non-player objects. Also, stable objects can emit a sound that indicates a standstill.

The player identifies the object on the screen from the sound by remembering the sound effect and associating it with the objects in the game, which is similar to the ordinary play style of blind gamers. The interaural level difference and acoustic volume indicate the horizontal and vertical relationships between the player character and objects. The change in interaural level differences and acoustic volume corresponds to the change of horizontal and 


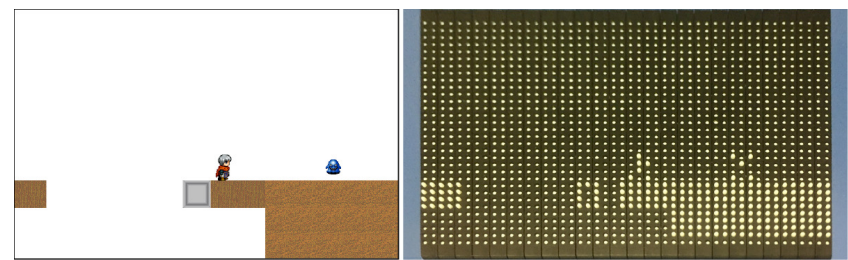

Figure 2: Graphical (left) and tactile (right) game views

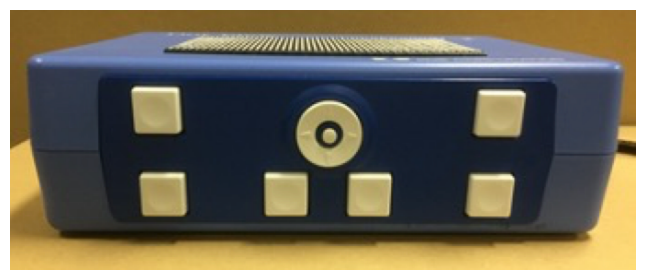

Figure 3: Controller of dot matrix display

vertical distances set to $\geq 2 \mathrm{~dB}$ and $\geq 1 \mathrm{~dB}$, respectively, from the studies on these thresholds, [17, 28, 46].

We implemented a function of surround viewer that automatically notifies the player of the positions of the walls, steps to go up and down, and overhead scaffolds with a dedicated sound effect when the player character approaches such objects. The player can pause the game at any time and check the terrain and object information on the screen using the abovementioned functions.

3.2.2 Touch play using dot-matrix display. For touch play, we prepared two displays that return tactile feedback, including a dotmatrix display (KGS DotView DV-2) and the tactile game controller named TactCon, having two tactile display modules at the shoulders.

From the left figure of Fig. 2, connecting the dot-matrix display to the computer makes operating the game possible by just touching the game screen. All screen information is provided by binary means and it varies depending on the up or down location. This dot-matrix display shows the position of the game character with a repeated up and down motion of the pins and positions of enemy characters, and field objects with a constant up motion of the pins. The players can recognize such information in real-time by touching the dot-matrix display and control keys at the left, right, and front sides of the display shown in Fig. 3.

3.2.3 Touch play using tactile game controller (TactCon). The TactCon enables gamers to obtain real-time tactile feedback without interfering with the control operation. From the preliminary feedback by visually impaired gamers, we designed and developed this game control. Fig. 4 shows the appearance of the TactCon. It has the general button and stick sets of a commercial gamepad, including a set of four buttons with the right thumb, a directional pad for the left thumb, an analog joystick, and start/select buttons.

While this controller does not have shoulder buttons, it has tactile display modules at the shoulders that we developed, as shown in Fig. 6. The tactile display consists of a matrix of small vibrator rods (32 rods). The size of the pin matrix is approximately $10 \mathrm{~mm} \times 10$ $\mathrm{mm}$, similar to the size of a fingertip. The vibrating pins on the tactile display are arranged in the shape of a matrix at intervals of $2 \mathrm{~mm}$, similar to the dot-to-dot distance of a Braille character. The spaces between the pins are closed using our designed multi-layered bimorph piezoelectric elements, as shown in the right part of Fig. 6.

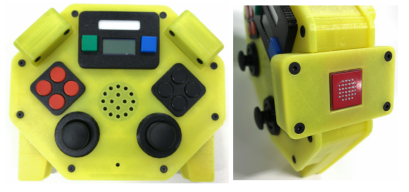

Figure 4: Tactile game controller TactCon
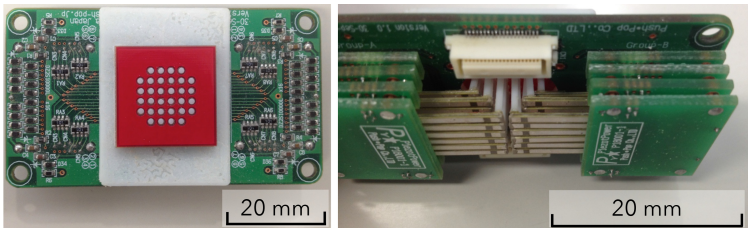

Figure 6: (Left) Top and (Right) side views of the tactile display module embedded to the TactCon shown in Fig.4.

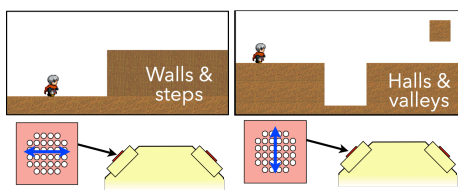

Left (presenting static stage situations)

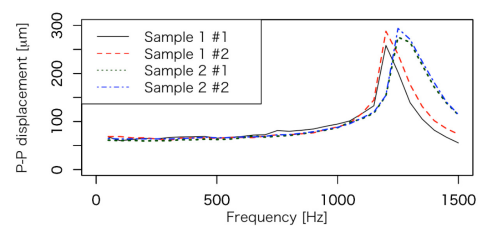

Figure 5: Displacement of the bimorph piezoelectric element versus frequency of applied voltage
Figure 7: Examples of the situations in the game and the corresponding tactile patterns presented by the TactCon

These pins are positioned in a $6 \times 6$ square, removing the pins at the four corners. Each pin vibrates individually at frequencies ranging from $50-400 \mathrm{~Hz}$ with proper timing and amplitude of more than 60 $\mu \mathrm{m}$ only by AC of $5 \mathrm{~V}$ (Fig. 5). Since the resonance frequency of the element was set at a high frequency of $1200 \mathrm{~Hz}$, power is transmitted well without the vibration of a pin becoming weak when a finger is applied. For the case of a lower resonance frequency (e.g., 400-500 $\mathrm{Hz}$ ), the pins' vibrations stops when the finger is put on the pins [18].

For a vibration sensation on the finger (mainly the index finger) using a tactile display, we adjusted the direction, speed, and interval of the vibrotactile sweep. Fig. 7 shows situations in the game and the implemented vibrotactile patterns corresponding to the situations. The left tactile display shows the existence of stable objects, such as walls and holes in the player's direction, and the right tactile display shows moving objects, such as flame, bullets, and enemy characters. This design enables easy mapping between the buttons and sticks on the left and right sides of the gamepad, which corresponds to the character's movement and fighting against enemies, respectively.

Table 1 shows the parameters of the direction, speed, and interval of the vibration sweeps in various game situations. The direction of the sweeps begins from where the game obstacle exists to where the player character stands. For example, a right-to-left sweep indicates that an obstacle is on the right of the player character. The sweep interval becomes shorter as the distance between the target obstacle and the player character approaches (number of blocks to the target $\times 500 \mathrm{~ms}$ ). We adjusted these parameters by several blind and sighted volunteers to make them discriminable.

3.2.4 Stage generation. Fig. 8 shows the stage elements used in the game. There are five categories of such elements, including walls 
Table 1: Correspondence between vibrotactile patterns and the obstacles to be presented in the game. Note that the blocks mean the relative distance between the player character and the target. One block corresponds to the size of the player character.

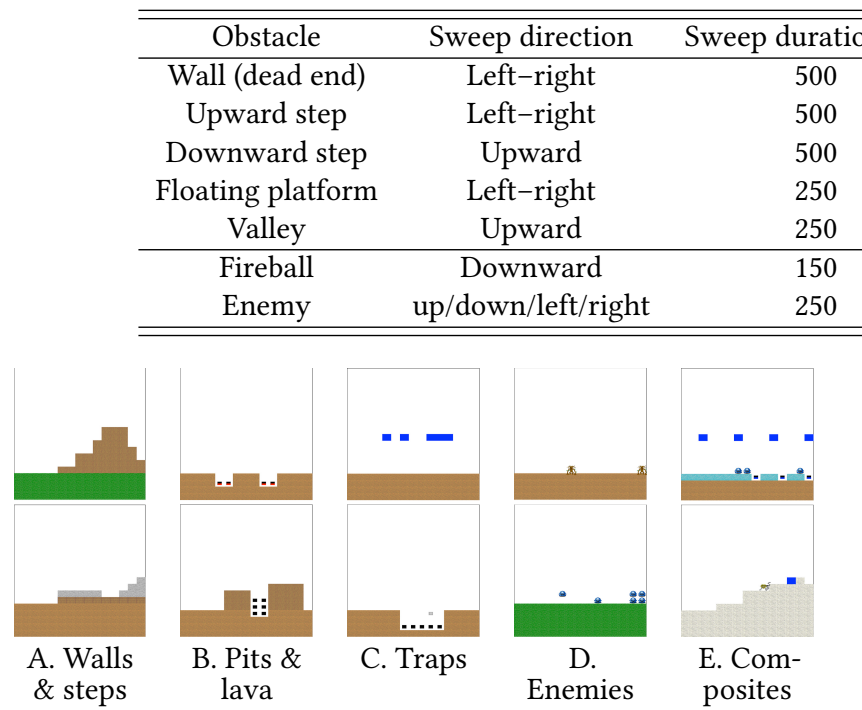

Figure 8: Examples of the stage elements.

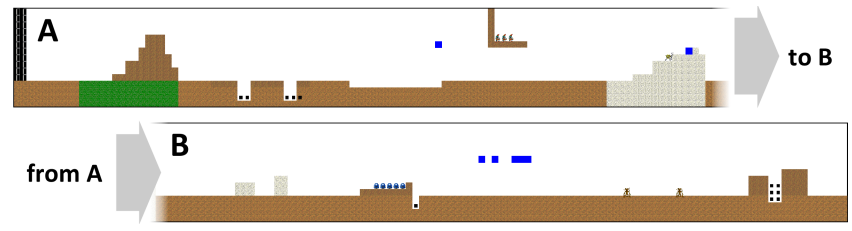

Figure 9: An example of the generated stage. This stage comprises from 10 stage elements schematically shown in Fig. 8.

\& steps, pits \& lava, traps, enemies, and composites. Categories A and $\mathrm{B}$ contain static objects, and $\mathrm{C}$ and $\mathrm{D}$ are composed of dynamic objects as well as static objects. Category E is composed of two substages of categories A D. Each category of the stage elements has four types, totaling 20 elements. Also, these four types have indices of 1-4: Earlier and later indices are relatively easy and difficult, respectively, according to our preliminary evaluation.

Fig. 9 shows an example of the generated stage, which is produced using the random allocation of 10 stage elements. The length of the stage is variable. For generating a stage with a length of 20 or fewer stage elements, the stage generator uses each stage element once. When the length of a stage exceeds 21 substages, the generator employs each stage element at least once. There are intervals whose horizontal length is as much as 5 units of the player character between the stage elements.

\section{EVALUATION}

We exhibited our inclusive action game for sighted and visually impaired people to play at two events, including the two-month exhibition at a museum. We designed the game so that visitors can select the multimodal play in the first half of the stage as a tutorial, and audio or touch plays in the second half of the stage (Fig. 10). In the latter part of the stage, visitors played the game without watching their game situation. The analysis was conducted using the play log acquired at the exhibitions. Our approved evaluation

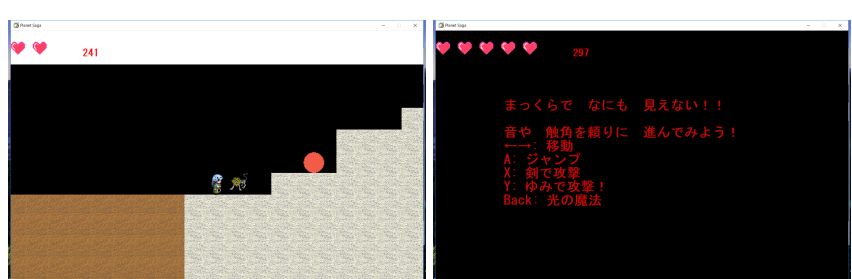

Figure 10: Example of a game screen. Left: Screen for multimodal play in the first half. Right: Dark screen in the second half. This screen only displays the instructions, including how to control player character.

was obtained from the office for Life Science Research Ethics and Safety at the University of Tokyo.

\subsection{Participants}

There were 388 participants, 211 males, 158 females, and 19 were unanswered. Regarding disability conditions, 322 sighted, 13 blind, and 8 low vision played our game. In the second half of the game, 202,84 , and 86 participants selected audio play, touch play with dot-matrix display, and touch play with TactCon, respectively. All of them were not hearing impaired.

\subsection{Procedure}

First, participants selected the difficulty level (beginner, intermediate, or advanced) of the game, and then confirmed the sound and vibration patterns and operation methods in the tutorial. Next, they experienced an overview of the game rules and auditory/tactile effects through the multimodal play mode that allows the visual, audio, and touch (the left figure of Fig. 10) as a tutorial. After watching the screen with the effect that the screen gradually darkened, the participants proceeded to the second half of the gameplay.

When the second half of the gameplay started, they selected one of the following play styles: audio play, audio and touch play using dot-matrix display, or audio and touch play using TactCon. Then, they played using the view of the right figure in Fig. 10. Simultaneously, they use "light magic" to brighten the screen for a few seconds as a relief measure during darkness. Also, the game recorded the playtime and number of keystrokes, including light magic, after obtaining the consent of the participants.

After reaching the goal, the participants took a voluntary questionnaire regarding their gaming experience, their impression of the game, and game effects. The 5-point Likert scale was used to gather responses to the questionnaire on impression. The questionnaire contained recognizable evaluations for auditory and tactile 
effects concerning the distance to walls and steps, the distance to and size of holes and valleys, movement of moving scaffolds and flame bullets, the position and movement of monsters, and the distance to objects in the horizontal and vertical directions. After that, the participants provided their overall score for the game.

\subsection{Analysis}

We obtained 253 valid play logs for the latter half after omitting the plays interrupted in the middle and those where participants used "light magic" over 10 times in the darkness and the number of keystrokes got more than 100 . The breakdown of valid data logs includes the plays by 125 males, 90 females, and the unanswered others. These participants included 184 sighted, 11 blind, 5 low vision, and other unanswered people. The number of play styles selected in the darkness was 118 for audio play, 59 for touch play with dot-matrix display, and 48 for touch play with TactCon.

From the obtained log data, we divided the log data in the gameplay during darkness into different play styles and compared the number of players cleared, playtime, number of damages taken, etc. The number of cleared players was analyzed using the $\chi^{2}$ test, with a cross table for each interface. To evaluate the elapsed time, total keystrokes, and total damages, we used the analysis of variance (ANOVA) to examine the significant main effects for user interfaces and participant characteristics. When the evaluated scales were non-normally distributed, aligned rank transform (ART) [45] was conducted on the scales before ANOVA. Also, we calculated the effect sizes, including Crámer's $V$ and partial $\eta^{2}$ and then, judged the degree of the effect size using the Cohena's indices criterion [13]. Then, the significance of the main effects was determined using posthoc multiple comparison methods from the least square means and Tukey's multiplicity adjustment [20,21].

Regarding responses to the subjective feedback, multiple ordinal logistic regression (OLR) [26] was employed to examine the significant contribution ratio of each participant attribute, such as visual condition, play style, game experience, and whether they cleared. The log odds ratio of each attribute was calculated to determine the degree of relevance of the responses. Next, the effect of the factors was discussed from the presence or absence of significant differences and the magnitude of the log odds ratio. The effect of the factor is greater than other factors when the log odds ratio of a factor was greater than 0 , and vice versa.

A series of analyses was conducted using R 4.0.2-4.0.3. We employed the packages, including psych, tidyverse, ARTool, and emmeans to conduct various statistical analyses.

\section{RESULTS AND DISCUSSION}

\subsection{Overview}

During the first half of multimodal play, 196 of 253 participants reached the goal (clear rate $77.4 \%$ ). Of those who could not, 26 exceeded the time limit, 3 lost because the health of their character became zero, and 28 stopped midway. During the darkness of the latter half of the game, 139 participants reached the goal (clear rate $54.9 \%$ ), 45 exceeded the time limit, 11 lost, and 30 players stopped midway.

From the results of ANOVA with ART for the clear rates of the stages, playstyle $\left(p=.018<.05\right.$, partial $\eta^{2}=.126$ (medium) $)$ and
Table 2: Cleared cases and rates by play styles in the visually impaired

\begin{tabular}{|c|c|c|c|c|}
\hline & $\begin{array}{l}p<0.01: * * \\
p<0.05: * \\
p<0.10:{ }^{*}\end{array}$ & $\begin{array}{c}\text { Audio\&Touch } \\
\text { play (DV2) }\end{array}$ & $\begin{array}{c}\text { Audio\&Touch } \\
\text { play (TactCon) }\end{array}$ & Audio play \\
\hline \multirow{3}{*}{ 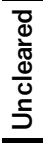 } & \# cases $(\%)$ & $3(75.0 \%)$ & $0(0 \%)$ & $1(12.5 \%)$ \\
\hline & Adjusted residual & 2.667 & -1.333 & -1.155 \\
\hline & $p$ & $0.008(* *)$ & 0.182 & 0.248 \\
\hline \multirow{3}{*}{$\begin{array}{l}\text { ठ } \\
\frac{\mathbb{J}}{0} \\
\frac{\mathbb{U}}{U}\end{array}$} & \# cases (\%) & $1(25.0 \%)$ & $4(100 \%)$ & $7(87.5 \%)$ \\
\hline & Adjusted residual & -2.667 & 1.333 & 1.155 \\
\hline & $p$ & $0.008(* *)$ & 0.182 & 0.248 \\
\hline
\end{tabular}

Fisher's test: $p=0.059(+)$, Crámer's $V: 0.677$ (Large)

Table 3: Cleared cases and rates by play styles in the sighted

\begin{tabular}{|c|c|c|c|c|}
\hline & $\begin{array}{l}\mathrm{p}<0.01: * * \\
\mathrm{p}<0.05: * \\
\mathrm{p}<0.10:{ }^{+}\end{array}$ & $\begin{array}{c}\text { Audio\&Touch } \\
\text { play (DV2) }\end{array}$ & $\begin{array}{c}\text { Audio\&Touch } \\
\text { play (TactCon) }\end{array}$ & Auc \\
\hline \multirow{3}{*}{ 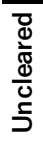 } & \# cases $(\%)$ & 4140.0 & $10(20$ & $34(36.2 \%)$ \\
\hline & Adjusted & 1.896 & -1.771 & -0.226 \\
\hline & $p$ & $0.058(+)$ & $0.077(+)$ & 0.821 \\
\hline \multirow{3}{*}{$\begin{array}{l}\bar{d} \\
\frac{d}{\mathbb{J}} \\
\frac{\mathbb{U}}{\cup}\end{array}$} & \# cases $(\%)$ & $26(52.0 \%)$ & $30(75.0 \%)$ & $60(73.8 \%)$ \\
\hline & Adjus & 189 & 1.771 & 0.226 \\
\hline & $p$ & $0.058(+)$ & $0.077(+)$ & 0.821 \\
\hline
\end{tabular}

$\chi 2$ test: $p=0.078(+)$, Crámer's $V: 0.166$ (Small)

frequency of game play ( $p<.001$, partial $\eta^{2}=.036$ (small)) had significant effects, while the disability condition had no significant effects $\left(p=.11>.10\right.$, partial $\eta^{2}=.033$ (small)). The result of ANOVA using the same factors with ART for the elapsed time indicated that there were significant main effects for the playstyle $\left(p<.001\right.$, partial $\eta^{2}=.091$ (medium)). Also, results for the total keystrokes under darkened conditions showed significant main effects only for playstyle $\left(p=.03<.05\right.$, partial $\eta^{2}=.030$ (small)).

\subsection{Game play on visually impaired players}

Table 2 shows the clear and unclear rates for each playstyle in visually impaired participants. From the $p$ values calculated using adjusted residuals, the players with TactCon and audio play significantly achieved a higher clearing rate than those with the dot-matrix display DV2. The participants who employed touch play with DV2 found it difficult to understand the consistency of the tactile and auditory presentation content. They stopped playing and checked the correspondence between the screen and the auditory information by touching the screen on the point map display with both hands, which made the game more complicated. The clearing time was significantly longer for reaching the goal using DV2 than in the other playstyles.

Some players who used TactCon mentioned that the simultaneous presentation of sounds and tactile senses increased the cues of in-game objects, making it easier to grasp the game situation. From visually impaired participants who were used to playing audio games, it was easier to use TactCon because the information in the game was distributed between auditory and tactile senses. Some mentioned that they were pleased the game sound was closer 
to that of commercial games because the system sound, added to convey the situation in the game during audio play, was eliminated. However, they stated that their fingertips got tired after touching the vibrating pins for a long time. These opinions suggest that immediate auditory and tactile presentation is important for gameplay in visually impaired players.

\subsection{Game play on sighted players}

Table 3 shows the clear and unclear rates for each playstyle in sighted participants. From the $p$ values calculated using adjusted residuals, the players using TactCon and DV2 significantly achieved higher or lower clearing rates than those using sound play. The same tendency was observed in the sighted participants as with the visually impaired. However, for the case of using DV2, the clearing rate was higher in the sighted than the visually impaired. Figures 11 and 12 show the results of audio/tactile feedback on the comprehensibility of events in the game for the sighted participants who did or did not complete the game. First, those who cleared the game rated all the items No. 1-6 significantly higher than those who had not cleared the game $\left(p=.0001\right.$, partial $\eta^{2}=.098$ (medium)). Moreover, in the evaluation scores of those who cleared the game, audio \& touch play with DV2 and TactCon were rated significantly higher than audio play $\left(p=.0001\right.$, partial $\eta^{2}=.015$ (small)).

DV2, which had a low clear rate, was rated as easier to understand than audio play because it was possible to touch the positional relationships between the player character and various objects displayed on the two-dimensional plane on the dot-matrix display. Furthermore, for the audio \& touch play with TactCon, the tactile information by the index finger solely can guess the situation in the game screen, which was ambiguous in audio play alone.

The subjective understandability shown in Fig. 12 indicates no significant difference between the evaluation items and the playstyle. However, the box plot in this figure shows that TactCon was rated the worst, while audio play was rated the highest. This is because the sighted participants, who did not complete the game, were not good at using tactile information. Some of the sighted participants reported that they did not understand the sweeping vibration at TactCon except for the finger they perceived the vibration. They stated that it was difficult grasping the direction of the vibration sweeping patterns with their fingertips when playing TactCon since they were not accustomed to tactile sensing. However, no difference exists between those who finished and could not finish the game in the auditory information, especially in the sense of distance (items 1 and 2). Though auditory information was presented in TactCon and DV2, we assumed that those who could not complete the game cannot associate the contents of the auditory and tactile presentations further.

\subsection{Helping factors of play}

From the results of the OLR in the clear rates of the stages with subjective understandability shown in Fig.11, the positions and behavior of monsters (No. 4$)$ were significant $(p=.048<.05)$. Additionally, the total elapsed time was mostly affected by the distance of the objects placed at the upper or lower parts of the player character (No. 4) $(p=.061<.10)$. When the participants understood the distance of the walls and steps in the game (No. 1), the total keystroke significantly decreased $(p<0.001)$.

For action games, such as this study, the degree of contribution was different for the presentation elements related to the game progress and those related to whether the player completed the game. Nevertheless, considering how the presentation of moving objects is understood in an inclusive action game was important.

\subsection{Overall evaluations}

From the item 7 in Fig. 11, most sighted participants who cleared the game scored 5 and almost all players using TactCon scored 5. This result indicates that the game, originally designed for the visually impaired, was enjoyable for the sighted players using the multimodal presentation with real-time tactile feedback. However, from the counterpart feedback shown in Fig. 12, we consider designing a method of initiation for those who could not use the tactile information or combining tactile and audio information smoothly to practice information detection.

\subsection{Limitations}

To collect play logs from several people, we evaluated the inclusive game at an exhibition and reported the existence of some sighted people who play games using audio and tactile presentations only. However, because the evaluation environment was not strictly controlled, it is necessary to conduct a controlled lab study to investigate the details. The tactile presentation method implemented in this study was determined from a preliminary study with some visually impaired and sighted people, so it may have been difficult for some players to perceive and understand or not have ensured immediacy. Additionally, the ease of playing the game differed depending on the experience of gameplay and braille reading experience, not to mention the tactile presentation. By verifying these points through control experiments, designing more inclusive game content and interfaces is possible as well as providing feedback for designing applications that can be enjoyed and operated regardless of visual impairment.

While the game was on exhibit, we prepared sub-monitors to display the visual game screen informing the surrounding spectators of the status of the game during the dark period. This setup enabled the players and the surrounding spectators to enjoy the game together. However, we did not control the situation where spectators and players enjoyed the game simultaneously, nor did we control the influence of spectators on players. Thus, such effects can affect the behavior of the players.

Although we could not confirm the behavior of all participants in this study, we found that some children gave up playing with the TactCon because of its large size and heaviness and then, switched to playing with typical commercial controllers. To design and investigate tactile experiences capable of attracting people of various backgrounds, developing a small and light controller with a tactile experience that children can easily operate is necessary.

\section{CONCLUSION}

This paper investigated the requirements for an inclusive action game that visually impaired and sighted players can play using audio and tactile information. First, we developed a 2D side-scrolling 


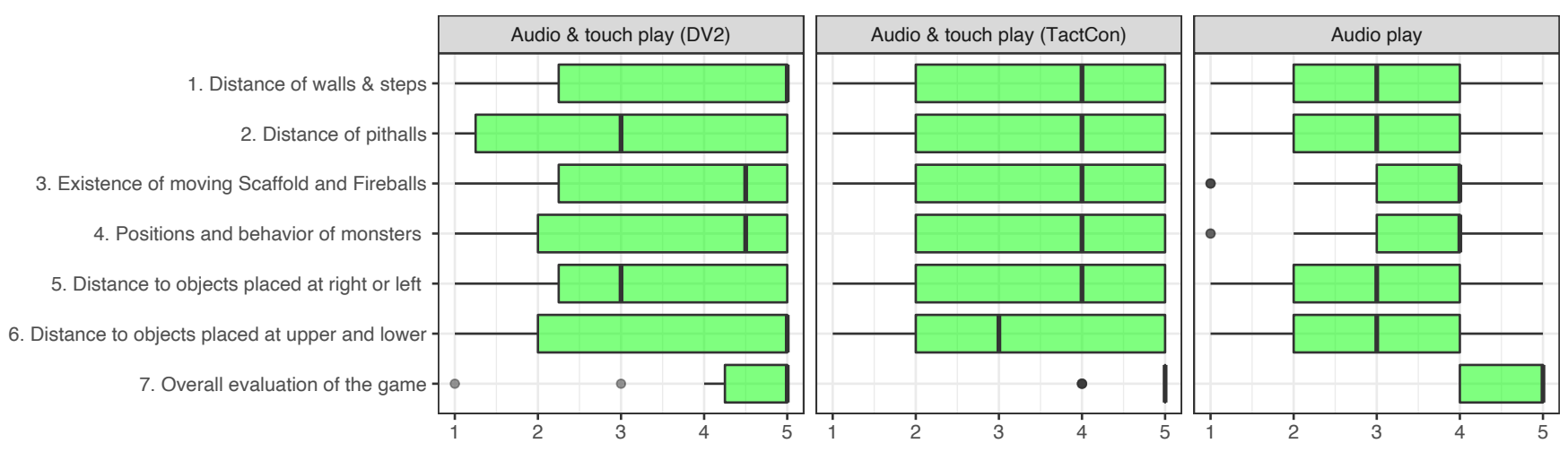

Figure 11: Subjective understandability on audio/tactile presentations of game objects in the sighted participants who cleared the game

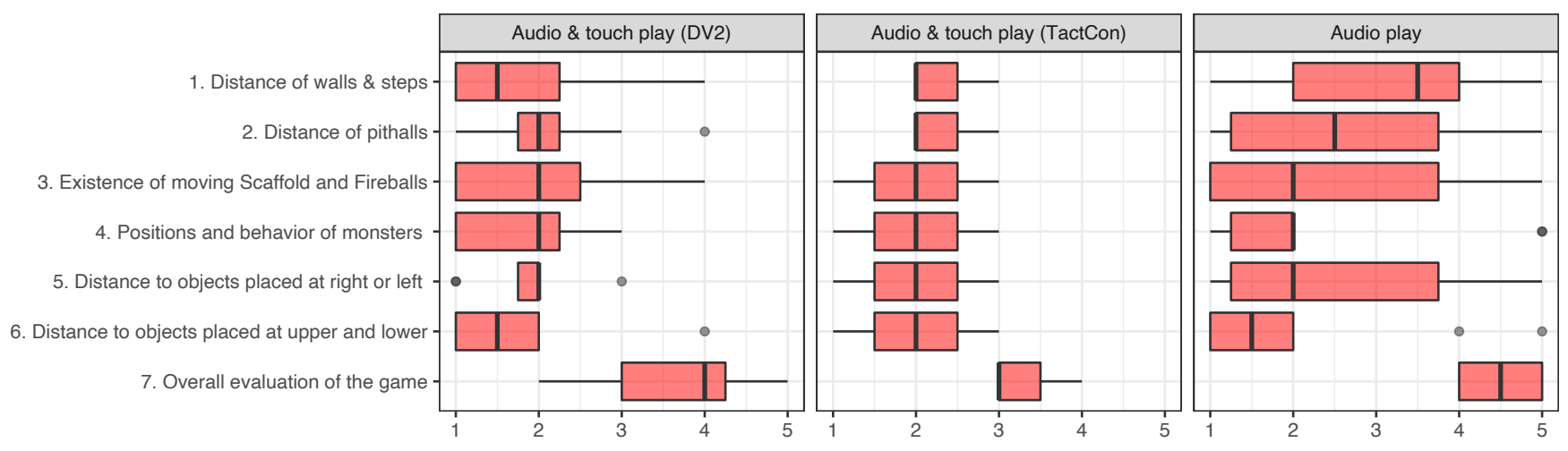

Figure 12: Subjective understandability on audio/tactile presentations of game objects in the sighted participants who did not clear the game

action game that enables gamers to manipulate a player character with auditory and tactile feedback. Then, we conducted a twomonth field evaluation of the game at an exhibition for collecting the play logs and impressions by visually impaired and sighted people.

The achievement of this study can be summarized as follows.

A1. Our results affirm that immediate auditory and tactile presentation can be important for visually impaired people to play action games requiring real-time response. A gamepad using tactile displays on both shoulders, as designed in this study, is an example of a gamepad that meets these requirements.

A2. When sighted persons play an action game only with sound and touch, it was suggested that they can complete the game by providing tactile support for parts that are ambiguous using audio information solely. However, because some sighted people are not accustomed to the tactile presentation and have difficulty in mapping the contents of the auditory and tactile presentations, we develop a method for streamlining the practice to perceive audio-tactile information and predict game situations.

A3. For action games, the helping factors for the players were different for presentation elements related to game progress and those related to whether the player can complete the game.
Nevertheless, it is important to consider how the presentation of moving objects is understood in an inclusive action game.

Our future work are as follows:

- Conducting controlled experiments about multimodal presentations on action games to investigate detailed effective parameters on e.g., vibrotactile patterns.

- Investigating the influence of spectators on the player's behavior and the effective settings to construct a good relationship or collaboration between them.

- Designing tactile experiences that children can play.

- Designing and developing other genres of multimodal inclusive games for persons with various visual conditions.

\section{ACKNOWLEDGMENTS}

This work was supported by JSPS KAKENHI Grant Numbers JP18H01047, JP18H01038, JP18J23363, JP20H04561, JP21H00885, and JP21K19782. We also thank Yuya Otsuka, Soichiro Yonemura, and Kiyotaka Nakao for the Planet Saga Development Team. We would also like to thank Mr. Tsuguchi of the Tomonotsu-Museum for inviting us to the exhibition. For the character voices, we received help from voice actors who are active on the Internet. We thank them for this. 


\section{REFERENCES}

[1] [n.d.]. Accessible game wiki for blind gamers. https://mm-galabo.com/ AcGameWiki/.

[2] [n.d.]. Audiogame.net. https://www.audiogames.net/.

[3] [n.d.]. BBC GEL / How to design accessible games. https://www.bbc.co.uk/gel/ guidelines/how-to-design-accessible-games.

[4] [n.d.]. Game accessibility guidelines. http://gameaccessibilityguidelines.com/.

[5] [n.d.]. Newzoo Global Games Market Report 2020. https://newzoo.com/insights/ trend-reports/newzoo-global-games-market-report-2020-light-version/.

[6] [n.d.]. Ryo-Gei-Kan. http://www.eonet.ne.jp/ akiraworld0719/gindex.html.

[7] [n.d.]. U.S. average age of video gamers 2019 | Statista. https://www.statista.com/ statistics/189582/age- of-us-video-game-players-since-2010/.

[8] [n.d.]. Web Content Accessibility Guidelines (WCAG) 2.1. https://www.w3.org/ TR/WCAG21/.

[9] Ronny Andrade, Melissa J Rogerson, Jenny Waycott, Steven Baker, and Frank Vetere. 2019. Playing Blind: Revealing the World of Gamers with Visual Impairment. In Proceedings of the 2019 CHI Conference on Human Factors in Computing Systems. 1-14.

[10] Ronny Andrade, Melissa J Rogerson, Jenny Waycott, Steven Baker, and Frank Vetere. 2020. Introducing the Gamer Information-Control Framework: Enabling Access to Digital Games for People with Visual Impairment. In Proceedings of the 2020 CHI Conference on Human Factors in Computing Systems. 1-14.

[11] Dominique Archambault, Roland Ossmann, Thomas Gaudy, and Klaus Miesenberger. 2007. Computer games and visually impaired people. Upgrade 8, 2 (2007), 43-53.

[12] Jen Beeston, Christopher Power, Paul Cairns, and Mark Barlet. 2018. Accessible Player Experiences (APX): The Players. In International Conference on Computers Helping People with Special Needs. Springer, 245-253.

[13] Jacob Cohen. 1992. A power primer. Psychological bulletin 112, 1 (1992), 155.

[14] Leonardo Garcez, Marcello Thiry, and Anita Fernandes. 2020. Accessible Features to Support Visually Impaired People in Game Development:: A Systematic Literature Review of the last 15 years. In 2020 15th Iberian Conference on Information Systems and Technologies (CISTI). IEEE, 1-6.

[15] Kathrin Gerling, Kieran Hicks, Michael Kalyn, Adam Evans, and Conor Linehan. 2016. Designing movement-based play with young people using powered wheelchairs. In Proceedings of the 2016 CHI Conference on Human Factors in Computing Systems. ACM, 4447-4458.

[16] Kathrin M Gerling, Regan L Mandryk, and Michael R Kalyn. 2013. Wheelchairbased game design for older adults. In Proceedings of the 15th International ACM SIGACCESS Conference on Computers and Accessibility. ACM, 27.

[17] Ervin R Hafter, Raymond H Dye, John M Neutzel, and Howard Aronow. 1977. Difference thresholds for interaural intensity. The fournal of the Acoustical Society of America 61, 3 (1977), 829-834.

[18] Takeshi Homma, Shuichi Ino, Takashi Izumi, Hayato Kuroki, and Tohru Ifukube. 2003. Measurement of mechanical characteristics of a fingerpad surface in the design of a tactile display. Journal of Robotics and Mechatronics 15, 2 (2003), 153-163.

[19] Akio Honda, Hiroshi Shibata, Jiro Gyoba, Kouji Saitou, Yukio Iwaya, and Yôiti Suzuki. 2007. Transfer effects on sound localization performances from playing a virtual three-dimensional auditory game. Applied Acoustics 68, 8 (2007), 885-896.

[20] Russell Lenth, Henrik Singmann, Jonathon Love, Paul Buerkner, and Maxime Herve. 2018. Emmeans: Estimated marginal means, aka least-squares means. $R$ package version 1, 1 (2018), 3.

[21] Russell V. Lenth. 2016. Least-Squares Means: The R Package lsmeans. Fournal of Statistical Software 69, 1 (2016), 1-33.

[22] N Lessard, M Pare, F Lepore, and M Lassonde. 1998. Early-blind human subjects localize sound sources better than sighted subjects. Nature 395, 6699 (1998), 278-280. https://doi.org/10.1038/26228

[23] Masaki Matsuo, Takahiro Miura, Masatsugu Sakajiri, Junji Onishi, and Tsukasa Ono. 2016. Audible Mapper \& ShadowRine: Development of Map Editor Using only Sound in Accessible Game for Blind Users, and Accessible Action RPG for Visually Impaired Gamers. In Computers Helping People with Special Needs, LNCS, Vol. 9759. Springer, 537-544.

[24] Masaki Matsuo, Takahiro Miura, Masatsugu Sakajiri, Junji Onishi, and Tsukasa Ono. 2017. Inclusive Side-Scrolling Action Game Securing Accessibility for Visually Impaired People. In IFIP Conference on Human-Computer Interaction. Springer, $410-414$.

[25] Masaki Matsuo, Masatsugu Sakajiri, Junji Onishi, Tsukasa Ono, and Takahiro Miura. 2017. Experience Report of a Blind Gamer to Develop and Improve the Accessible Action RPG ShadowRine for Visually Impaired Gamers. Fournal on Technology and Persons with Disabilities (2017).

[26] Peter McCullagh. 1980. Regression models for ordinal data. Journal of the Royal Statistical Society: Series B (Methodological) 42, 2 (1980), 109-127.

[27] Klaus Miesenberger, Roland Ossmann, Dominique Archambault, Gig Searle, and Andreas Holzinger. 2008. More than just a game: accessibility in computer games. In Symposium of the Austrian HCI and Usability Engineering Group. Springer, 247-260.
[28] George A Miller. 1947. Sensitivity to changes in the intensity of white noise and its relation to masking and loudness. The fournal of the Acoustical Society of America 19, 4 (1947), 609-619.

[29] Takahiro Miura, Tohru Ifukube, and Shigeto Furukawa. 2011. Contribution of acoustical characteristics to auditory perception of silent object. In Systems, Man, and Cybernetics (SMC), 2011 IEEE International Conference on. IEEE, 1074-1079.

[30] Takahiro Miura, Masaki Matsuo, Ken-ichiro Yabu, Atsushi Katagiri, Masatsugu Sakajiri, Junji Onishi, Takeshi Kurata, and Tohru Ifukube. 2020. GAME: Game As a Measurement Environment: Scheme to Evaluate Interfaces and Game Contents Based on Test Theories. Proceedings of the ACM on Interactive, Mobile, Wearable and Ubiquitous Technologies 4, 4 (2020), 1-30.

[31] Takahiro Miura, Teruo Muraoka, and Tohru Ifukube. 2010. Comparison of obstacle sense ability between the blind and the sighted: A basic psychophysical study for designs of acoustic assistive devices. Acoustical Science and Technology 31, 2 (2010), 137-147.

[32] Tony Morelli, John Foley, Luis Columna, Lauren Lieberman, and Eelke Folmer. 2010. VI-Tennis: a vibrotactile/audio exergame for players who are visually impaired. In Proceedings of the Fifth International Conference on the Foundations of Digital Games. ACM, 147-154.

[33] Tony Morelli, John Foley, and Eelke Folmer. 2010. Vi-bowling: a tactile spatial exergame for individuals with visual impairments. In Proceedings of the 12th international ACM SIGACCESS conference on Computers and accessibility. ACM, 179-186.

[34] Tony Morelli and Eelke Folmer. 2014. Real-time sensory substitution to enable players who are blind to play video games using whole body gestures. Entertainment Computing 5, 1 (2014), 83-90.

[35] Melanie Nind. 2014. What is inclusive research? A\&C Black.

[36] Makoto Ohuchi, Yukio Iwaya, Yôiti Suzuki, and Tetsuya Munekata. 2006. A comparative study of sound localization acuity of congenital blind and sighted people. Acoustical Science and Technology 27, 5 (2006), 290-293.

[37] John R Porter and Julie A Kientz. 2013. An empirical study of issues and barriers to mainstream video game accessibility. In Proceedings of the 15th international ACM SIGACCESS conference on computers and accessibility. ACM, 3.

[38] Kyle Rector, Cynthia L Bennett, and Julie A Kientz. 2013. Eyes-free yoga: an exergame using depth cameras for blind \& low vision exercise. In Proceedings of the 15th International ACM SIGACCESS Conference on Computers and Accessibility. ACM, 12.

[39] Didier Schwab, Amela Fejza, Loïc Vial, and Yann Robert. 2018. The GazePlay Project: Open and Free Eye-trackers Games and a Community for People with Multiple Disabilities. In 16th International Conference on Computers Helping People with Special Needs.

[40] Yoshikazu Seki and Norio Nakamura. 2000. Obstacle Sense in Sound Field with Plural Circumstance Noise. Transactions of the Virtual Reality Society of Japan 5 , 3 (2000), 989-995.

[41] Yoshikazu Seki and Tetsuji Sato. 2011. A training system of orientation and mobility for blind people using acoustic virtual reality. IEEE Transactions on neural systems and rehabilitation engineering 19, 1 (2011), 95-104.

[42] Matthew T Tull, Keith A Edmonds, Kayla M Scamaldo, Julia R Richmond, Jason P Rose, and Kim L Gratz. 2020. Psychological outcomes associated with stay-athome orders and the perceived impact of COVID-19 on daily life. Psychiatry research 289 (2020), 113098 .

[43] Gyoergy Wersenyi. 2012. Virtual localization by blind persons. Fournal of the Audio Engineering Society 60, 7/8 (2012), 568-579.

[44] Thomas Westin, JaEun Jemma Ku, Jérôme Dupire, and Ian Hamilton. 2018. Game Accessibility Guidelines and WCAG 2.0-A Gap Analysis. In International Conference on Computers Helping People with Special Needs. Springer, 270-279.

[45] Jacob O Wobbrock, Leah Findlater, Darren Gergle, and James J Higgins. 2011. The aligned rank transform for nonparametric factorial analyses using only anova procedures. In Proceedings of the SIGCHI conference on human factors in computing systems. 143-146.

[46] William A Yost and Raymond H Dye Jr. 1988. Discrimination of interaural differences of level as a function of frequency. The fournal of the Acoustical Society of America 83, 5 (1988), 1846-1851.

[47] Bei Yuan, Eelke Folmer, and Frederick C Harris. 2011. Game accessibility: a survey. Universal Access in the Information Society 10, 1 (2011), 81-100.

[48] BRANNON Zahand. 2007. Making video games accessible: business justifications and design considerations. MSDN Library Technical Articles. Accessed 6 (2007). 